\title{
Vehicle Electrification Impacts on Energy Consumption for Different Connected-Autonomous Vehicle Scenario Runs ${ }^{\dagger}$
}

\author{
Ehsan Sabri Islam *, Ayman Moawad, Namdoo Kim and Aymeric Rousseau \\ Argonne National Laboratory, 9700 South Cass Avenue, Lemont, IL 60439, USA; amoawad@anl.gov (A.M.); \\ nkim@anl.gov (N.K.); arousseau@anl.gov (A.R.) \\ * Correspondence: eislam@anl.gov; Tel.: +1-630-252-2841 \\ + This paper is an adaptation of the paper presented at the 32nd International Electric Vehicles Symposium and \\ Exhibition, Lyon, France, 19-22 May 2019.
}

Received: 18 October 2019; Accepted: 24 December 2019; Published: 27 December 2019

\begin{abstract}
Transportation system simulation is a widely accepted approach to evaluate the impact of transport policy deployment. In developing a transportation system deployment model, the energy impact of the model is extremely valuable for sustainability and validation. It is expected that different penetration levels of Connected-Autonomous Vehicles (CAVs) will impact travel behavior due to changes in potential factors such as congestion, miles traveled, etc. Along with such impact analyses, it is also important to further quantify the regional energy impact of CAV deployment under different factors of interest. The objective of this paper is to study the energy consumption of electrified vehicles in the future for different penetration levels of CAVs deployment in the City of Chicago. The paper will further provide a statistical analysis of the results to evaluate the impact of the different penetration levels on the different electrified powertrains used in the study.
\end{abstract}

Keywords: transportation system modeling; connected-autonomous vehicle (CAV); electric vehicle (EV); energy consumption; vehicle simulation

\section{Introduction}

Understanding the energy consumption of current and future vehicle technologies under real-world conditions is critical to estimating the overall impact of system models. Estimating the energy consumption during measured real-world drive cycles provides a good approximation, but does not ensure a consistent impact on the transportation system model as a whole [1]. This is why it is important to evaluate the energy impact on the drive cycles generated by the transportation system model itself.

The transportation system modeling tool, POLARIS [2,3] is used to develop and validate the transportation system model for different metropolitan cities of the United States. It uses population and vehicle synthesis, along with activity demand generation and traffic flow to model the system. An individual-level CAV technology choice framework is also implemented along with updated traffic flow modeling to account for CAVs. A study was implemented in POLARIS to analyze the impact of CAV technologies on travel demand and energy consumption in the City of Chicago.

The resulting stochastic speed profiles from POLARIS, combined with the data on driving cycles and fleet distribution are used as an input to Autonomie [4], a vehicle system modeling tool. Using the speed profiles, Autonomie was able to simulate the energy consumption of the transportation network for different vehicle technologies. Figure 1 illustrates the steps involved with the process. 


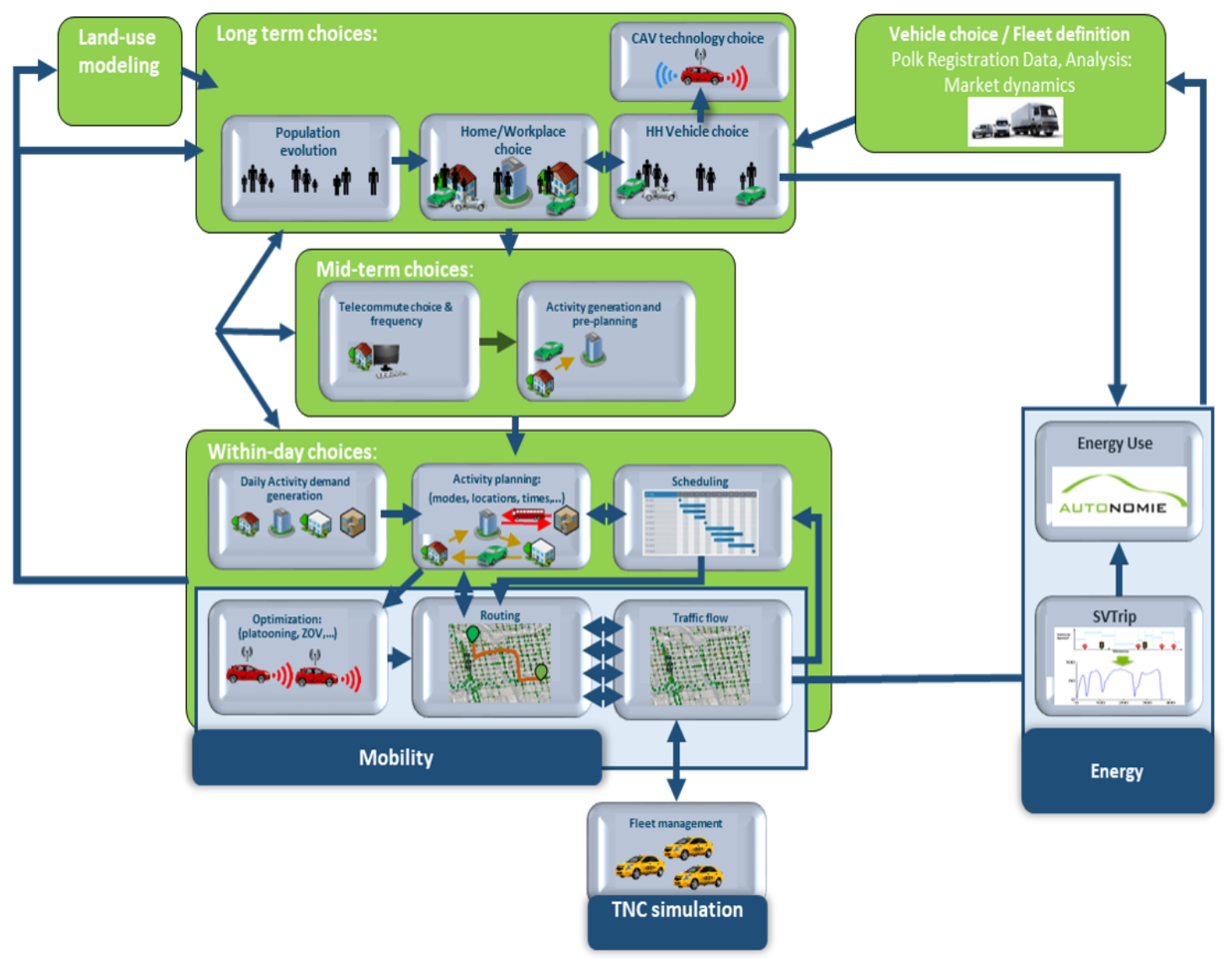

Figure 1. CAVs modeling using POLARIS \& Autonomie.

Previous studies demonstrated the importance and ability of evaluating the energy consumption of transportation system models in real world scenarios to analyze the intersection between transport policy and vehicle technology [5].

There are many traffic flow simulation tools that model CAV technologies as part of the U.S. Department of Energy Vehicle Technologies Office (DOE-VTO) Systems and Modeling for Accelerated Research in Transportation (SMART) Mobility Consortium. These models study the impact in mobility due to the introduction of CAVs through microsimulation traffic flow models, such as AimSun [6] and VisSim [7]; and mesoscopic traffic flow models such as POLARIS and BEAM [8]. There are differences in the assumptions and modeling techniques between these different transportation system tools. However addressing the assumptions of the different traffic flow models and their applications themselves is beyond the scope of this paper, which is to evaluate the energy consumption differences for one of the studies previously conducted in POLARIS.

The different literature studies and existing research in modeling the traffic flows for CAVs are discussed in greater detail in Auld et al., 2017 [9]. This paper only focuses on the energy consumption impacts of different vehicle powertrains for different CAV scenarios by isolating the impacts of vehicle electrification. Auld et al. also discusses the details in demand generation, traffic assignment and simulation of CAVs.

There were also several studies conducted to study the energy consumption of transportation system models using different methods. Colin et al. [8] models the charging behavior and infrastructure in BEAM. The study uses constant energy consumption unit values $(\mathrm{kWh} / \mathrm{km})$ for different average speeds in the traffic flow. However to model plug-in hybrids, there are a lot of instantaneous control behaviors (such as state-of-charge of batteries, etc.) that need to be accounted for when evaluating the energy consumption. Hence U.S. DOE-VTO supports using Autonomie, a full vehicle simulation tool to evaluate the energy consumption for transportation system simulation tools. Many projects funded 
through U.S. DOE-VTO are currently implemented using Autonomie for different transportation system simulations as it accurately represents the energy consumption impacts of the traffic flow. Autonomie further implements U.S. DOE-VTO technology targets for vehicle components (such as engine efficiency, battery specific energy, etc.) [10] and therefore, would be applicable in evaluating the impact of overall VTO portfoliio.

There are also several studies analyzing the potential impact of cooperative and adaptive cruise control (CACC), a key CAV technology, on traffic flow and energy consumption. According to Christopher et al. [11], CACC could lead to a following headway of 0.6 seconds. This would reduce the drag forces and decrease energy consumption. Analysis of field data shows $14 \%$ savings in fuel consumption of trucks [12]. Under different test environments, other studies estimate the reduction in trucks' fuel consumption between $10 \%$ and $21 \%$ [13], $8 \%$ and $11 \%$ [14], and $5 \%$ and $8 \%$ [15]. Similar studies performed on light duty vehicles demonstrate varying fuel efficiency improvements [16-18]. However these studies had been conducted in specific test tracks within laboratory settings and do not replicate the impact in real world driving. Using the combination of POLARIS and Autonomie enhances the ability to evaluate the real-world impact in metropolitan cities.

\section{POLARIS}

POLARIS is an agent-based, activity-based model of integrated traveler behavior and traffic flow. In a POLARIS simulation, the population is synthesized and activities for each individual are generated and then planned using different heuristic methods combined with discrete choice models. The activity start time and schedules are subject to change as the simulation is running and traffic congestion is updated. The individual vehicles are moved through the links and nodes using a mesoscopic simulation approach, based on the Newell kinematic waves model [19] subject to speeds and capacities at the links, as well as turning and capacity constraints at the intersections. The simulation serves as a feedback mechanism by providing the network performance for the route choice model, the demand model, and the intelligent transportation systems (ITS) model. A set of measures of effectiveness (MOE) are produced such as average speed, density, flow rate, and vehicle trajectories.

As part of the developments within SMART Mobility Consortium, different cities within the U.S. are being developed in POLARIS. Currently, there are models for Southwest Michigan [5], Bloomington, IN and Chicago, IL. POLARIS is being developed through collaboration of researchers around the world [20,21]; however, the transportation system models currently implemented in POLARIS are limited to United States.

The Chicago Metropolitan Area was developed in POLARIS in collaboration with Chicago Metropolitan Agency for Planning (CMAP). Figure 2 illustrates a snapshot from the Chicago road network that was developed.

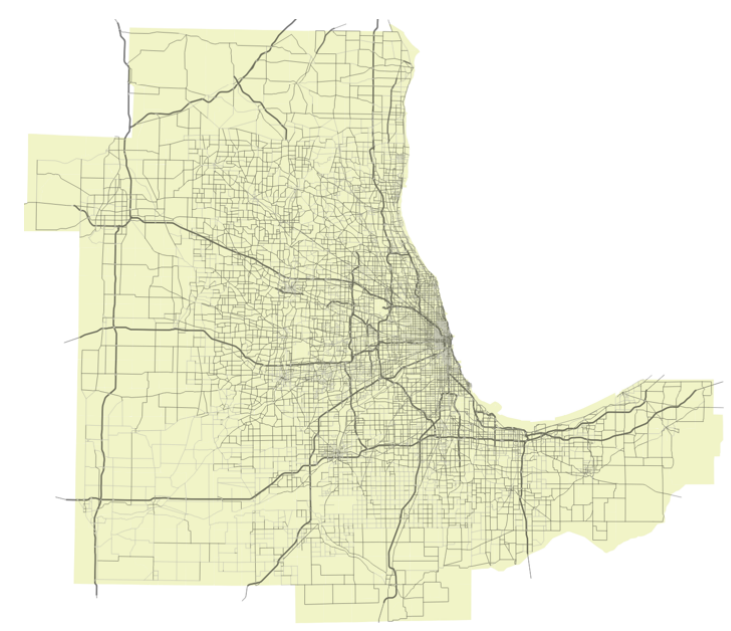

The model consists of:

- 10.2 million travelers

- 27.9 million automobile trips

- 31,278 links in 1944 zones for the 20 county region

Figure 2. Chicago Metropolitan Area Road Network. 
Three levels of CAV cost scenarios were setup to evaluate the impact of CAV penetration in the traffic flow. Here, the CAV cost refers to Willingness to Pay (WTP) for vehicle automation. There are multiple studies that model the WTP for vehicle automation through various surveys, modeling techniques and different variables used. Bansal et al. [22] addressees the survey conducted in Austin, Texas to study the WTP for vehicle automation. Shabanpour et al. [23] discusses the findings of these studies in further detail.

Table 1 details the different assumptions considered for the study. These assumptions were developed from various literature studies and were addressed in detail in Auld, 2017 [9].

Table 1. CAV case study setup.

\begin{tabular}{ccc}
\hline Case & CAV Cost (\$) (Willingness to Pay) & CAV Fleet Penetration (\%) \\
\hline 1 & 15,000 & 13.4 \\
2 & 5000 & 47.8 \\
3 & 0 & 100 \\
\hline
\end{tabular}

It can be seen that the penetration of CAVs increases significantly with decreasing cost of automation.

\section{Autonomie}

The Vehicle System Simulation tool Autonomie is used to perform simulations on drive cycles with the vehicle models that incorporate baseline and advanced vehicle technology targets as generated for U.S. DOE [10] and U.S. DOT [24]. The vehicle models used to evaluate the energy consumption on the drive cycles consist of gasoline conventional powertrains, power-split hybrid-electric vehicles (HEVs), plug-in HEVs and battery electric vehicles (BEVs) of different all-electric ranges (AERs). Multiple EPA class definitions of vehicles (Compact, Midsize, Midsize SUV and Midsize Trucks) have also been used to evaluate the energy consumption on the driving profiles. Market penetration models are used to select the advanced vehicle powertrain models for future years.

Table 2 details a subset of the different vehicle powertrains used to represent the fleets:

Table 2. Autonomie Vehicle Models Considered.

\begin{tabular}{ccc}
\hline Powertrain & \multicolumn{2}{c}{ Vehicle Technology } \\
\hline & Engine & Transmission \\
\hline CONV (Mild Hybrid 48V) & SkyActiv & 10-speed Automatic \\
Power-Split HEV & Power Split & \\
Voltec PHEV 50AER & Power Split & \\
BEV 200AER & & \\
\hline
\end{tabular}

The component and vehicle assumptions are derived from the U.S. Department of Energy Targets. Table 3 lists the detailed component-level assumptions. 
Table 3. Vehicle Component Assumptions.

\begin{tabular}{ccc}
\hline Component Assumption & Powertrain & Value \\
\hline \multirow{2}{*}{ Battery Specific Power $(\mathrm{W} / \mathrm{kg})$} & HEVs & 6000 \\
& PHEVs & 1500 \\
& BEVs & 870 \\
\hline \multirow{2}{*}{ Battery Energy Density $(\mathrm{Wh} / \mathrm{kg})$} & PHEVs & 188.89 \\
& BEVs & 340 \\
\hline \multirow{2}{*}{ Engine Efficiency (\%) } & CONVs & 38.78 \\
& HEVs & 52 \\
\hline Motor Efficiency $(\%)$ & BEV & 97 \\
\hline
\end{tabular}

\section{Simulation Results Analysis}

Figure 3a illustrates the percentage of fuel consumption (in $\mathrm{kg}$ ) change between the baseline case (with maximum CAV cost scenario of $\$ 15,000$ ) and case 3 (with no CAV cost) and Figure $3 \mathrm{~b}$ summarizes the different parameters of interest (vehicle miles traveled, average trip length, fuel consumption, etc.) across the three different CAV penetration levels. The detailed results of mobility changes across the different CAV penetration cases were presented in Auld et al., 2017 [9].

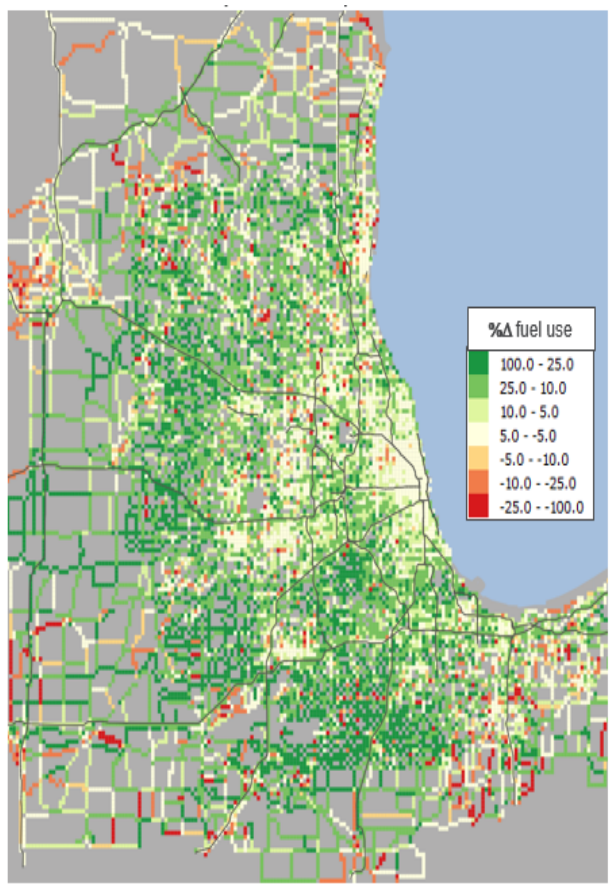

(a) Geographic distribution of \% fuel consumption changes between case 1 and 3

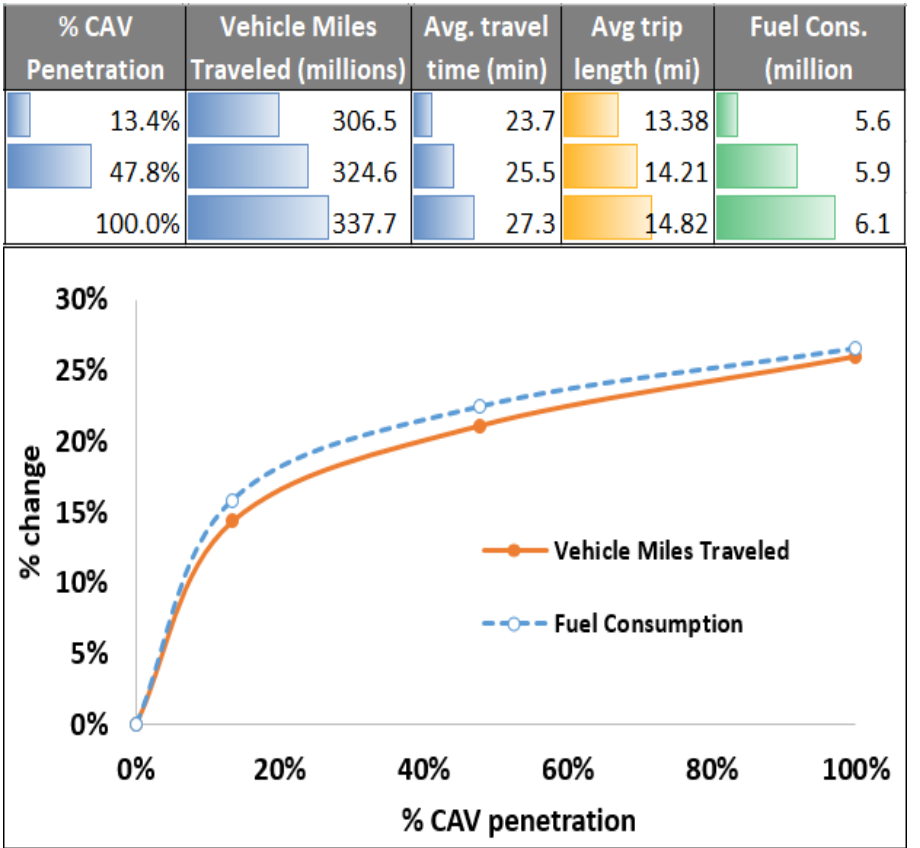

(b) Summary table of the different CAV penetration case runs

Figure 3. Summary of CAV study simulation run.

In Figure 3a, the dark green areas indicate higher total fuel consumption (in $\mathrm{kg}$ ) for the CAV cost case of $\$ 0$. This is because higher CAV fleet penetration results in increased traffic flow in urban areas, resulting in higher fuel consumption. However we observe a reduction in fuel consumption in the suburban areas. This is driven by how the traffic flow is generated due to the increased penetration of $\mathrm{CAVs}$ in the model. The Figure $3 \mathrm{~b}$ shows the changes in total fuel consumption (in $\mathrm{kg}$ ) and vehicle miles traveled (VMT) across the different CAV penetration levels. It shows that with increasing \%CAV penetration, the total fuel consumption (in $\mathrm{kg}$ ) increases much more aggressively compared to the VMT increments. 
This section further details the breakdown of impact observed in energy consumption of the different $\mathrm{CAV}$ cost scenarios across the different vehicle powertrains.

\subsection{Conventional Vehicle Powertrain}

Figure 4a illustrates the distribution of fuel consumption with respect to driving distance for conventional vehicles for the three different penetration levels of CAVs. Figure $4 \mathrm{~b}$ shows the box plot of fuel consumption of conventional vehicles for the three different penetration levels of CAVs.
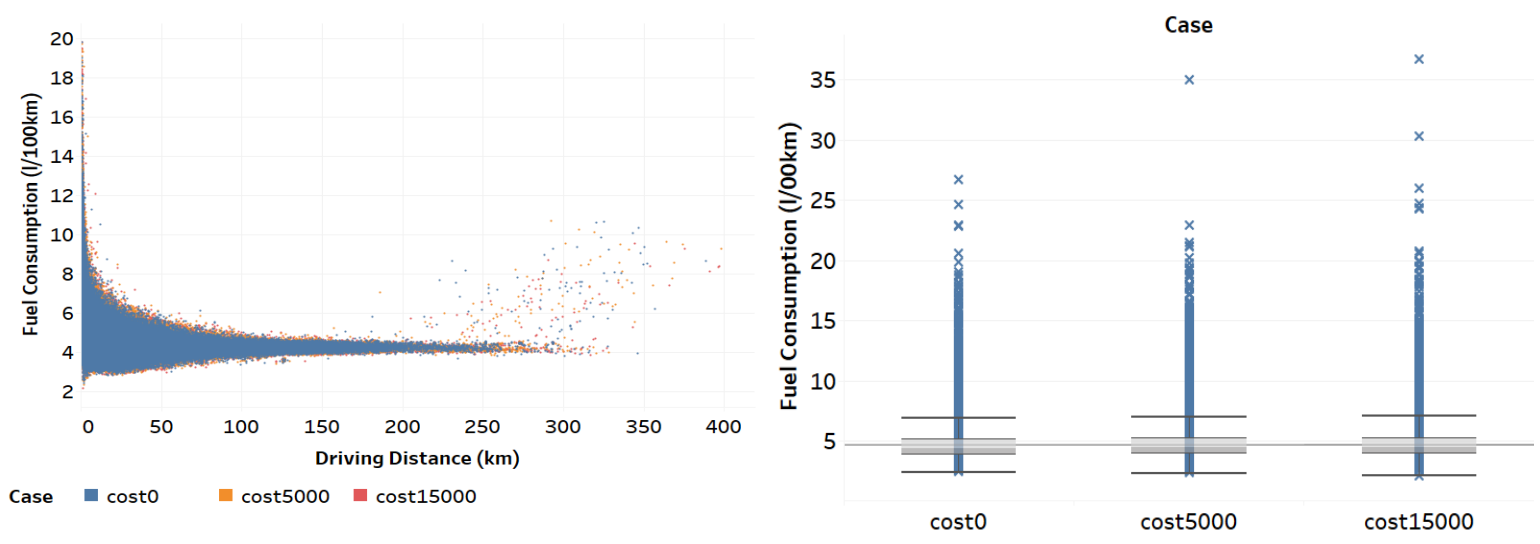

(a) Fuel consumption vs. driving distance of conventional vehicles

(b) Fuel consumption box-plot for conventional vehicles

Figure 4. Fuel consumption distribution of conventional vehicles.

From Figure $4 a$, it can be seen that with respect to the driving distances of individual trips, the fuel consumption rate $(\mathrm{L} / 100 \mathrm{~km})$ is very similar across the different scenarios. Figure $4 \mathrm{~b}$ further shows that the variation in average fuel consumption rates for conventional vehicles across the three different CAV cost scenarios is negligible, although the full extent of the ranges could vary. This is because the vehicle designs were indifferent for the different $C A V$ scenario runs and hence do not show any drastic difference across the different cases.

\subsection{Hybrid-Electric Vehicles (HEVs)}

Figure 5a illustrates the distribution of fuel consumption rate $(\mathrm{L} / 100 \mathrm{~km})$ with respect to driving distance for HEVs for the three different penetration levels of CAVs. Figure $5 b$ shows the box plot of fuel consumption of HEVs for the three different penetration levels of CAVs.

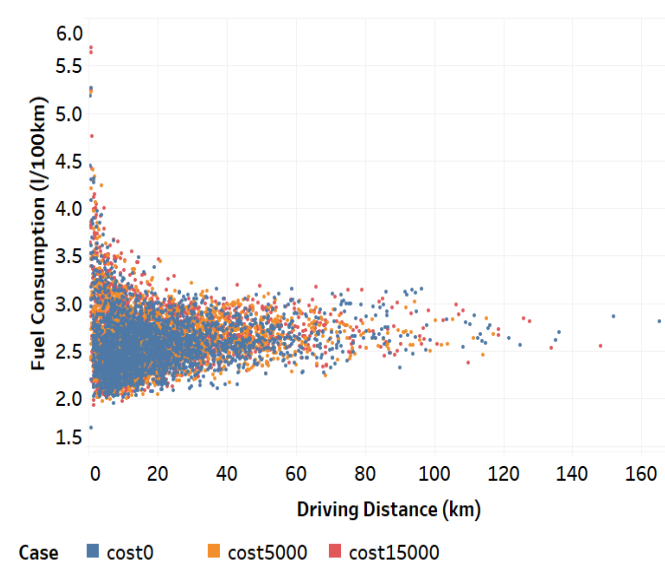

(a) Fuel consumption vs. driving distance of HEVs

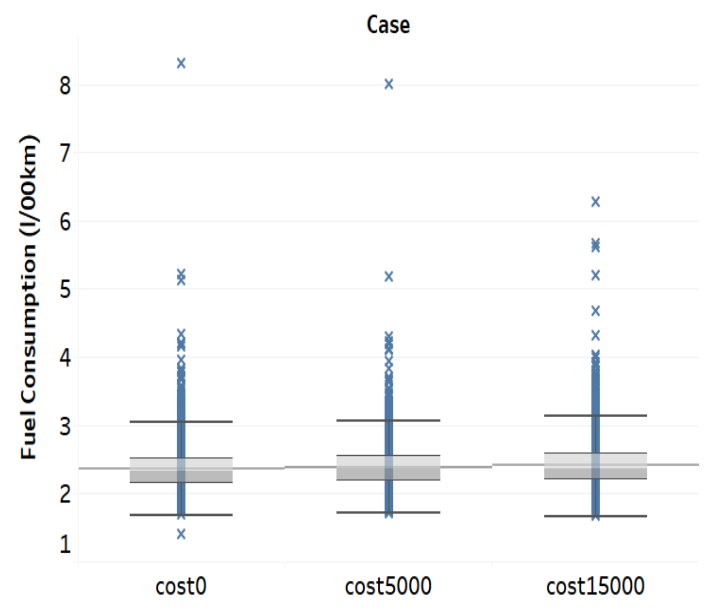

(b) Fuel consumption box-plot for HEVs

Figure 5. Fuel consumption distribution of HEVs. 
Similar to Figure $4 \mathrm{a}$, it can be seen that with respect to driving distance, the distribution of fuel consumption rate $(\mathrm{L} / 100 \mathrm{~km})$ for HEVs is very similar across the different cases in Figure $5 \mathrm{a}$. Figure $5 \mathrm{~b}$ further shows that the average fuel consumption rate $(\mathrm{L} / 100 \mathrm{~km})$ is similar across the different cases, although the full extent could vary due to different trips being affected through the traffic flow. For power-split HEVs, the fuel consumption is the driving factor and the impacts in electrical energy consumption is not as significant.

\subsection{Plug-In Hybrid-Electric Vehicles (PHEVs)}

Figure 6a illustrates the distribution of fuel consumption with respect to driving distance for PHEVs for the three different penetration levels of CAVs. Figure $6 \mathrm{~b}$ shows the box plot of fuel consumption of PHEVs for the three different penetration levels of CAVs.

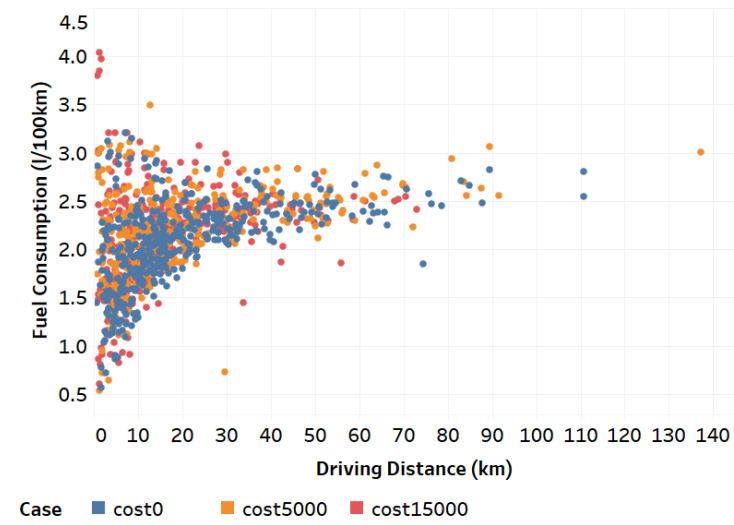

(a) Fuel consumption vs. driving distance for PHEVs

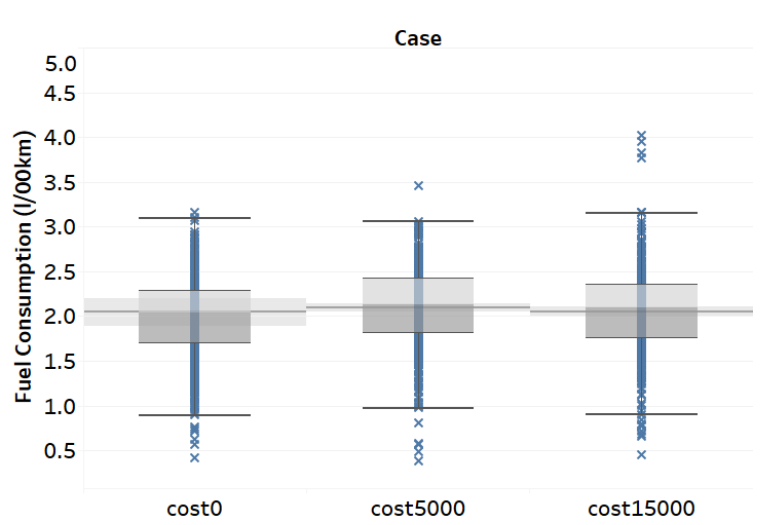

(b) Fuel consumption box-plot for PHEVs

Figure 6. Fuel consumption distribution for PHEVs.

Figure $6 \mathrm{a}, \mathrm{b}$ show that the fuel consumption rate (in L/100 km) is very similar across the different CAV cost scenarios. Figure 6a shows that the fuel consumption rates (in L/100 km) across driving distances of the various trips seem to not be influenced by the CAV cost scenarios.

Figure 7a illustrates the distribution of electrical energy consumption with respect to driving distance for PHEVs for the three different penetration levels of CAVs. Figure $7 \mathrm{~b}$ shows the box plot of fuel consumption of PHEVs for the three different penetration levels of CAVs.

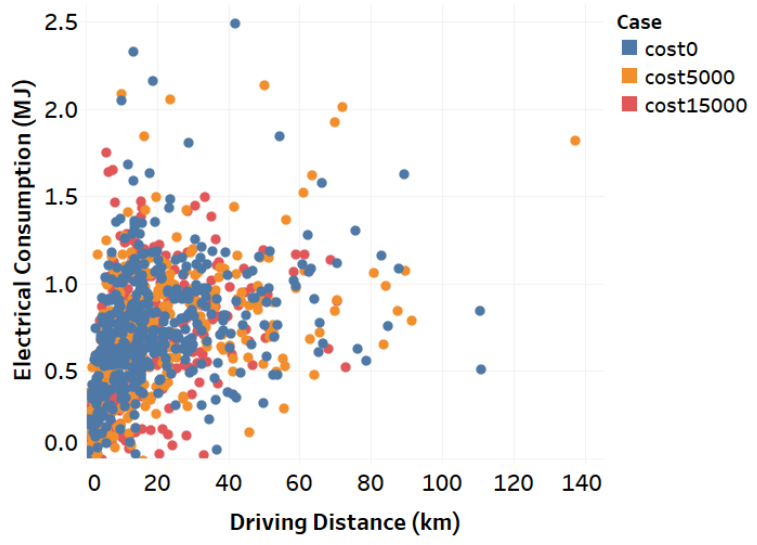

(a) Electrical consumption vs. driving distance for PHEVs

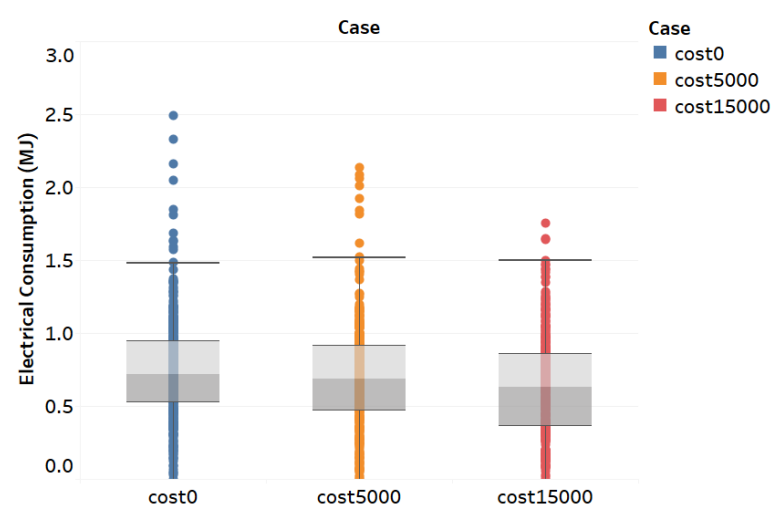

(b) Electrical consumption box-plot for PHEVs

Figure 7. Electrical consumption distribution for PHEVs.

From Figure $7 \mathrm{a}$, the variation in electrical energy consumption across driving distance is not apparent between the different CAV cost scenarios. However in Figure $7 \mathrm{~b}$, the variation in electrical 
energy consumption for the trips across the different CAV cost scenarios can be easily differentiated. Considering the full range of electrical energy consumption values, it seem to increase with lower CAV cost cases and hence higher CAV penetration in the traffic flow.

\subsection{Battery Electric Vehicles (BEVs)}

Figure 8a illustrates the distribution of electrical energy consumption with respect to driving distance for BEVs for the three different penetration levels of CAVs and Figure 8b shows the box plot of fuel consumption of BEVs for the three different penetration levels of CAVs.

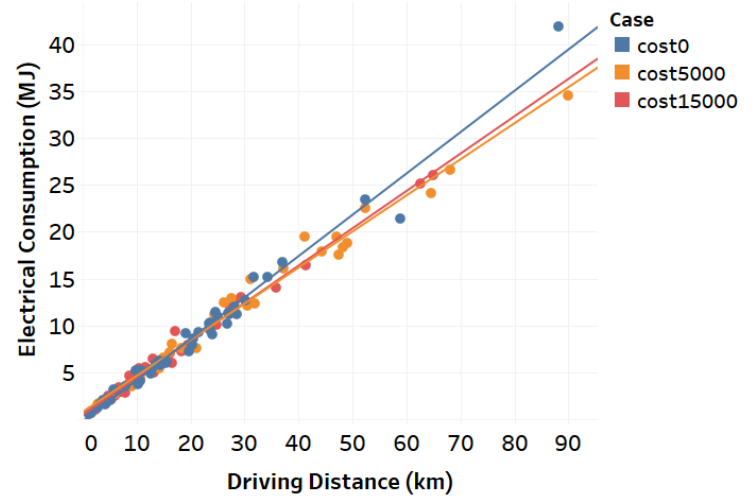

(a) Electrical Consumption vs. driving distance for BEVs

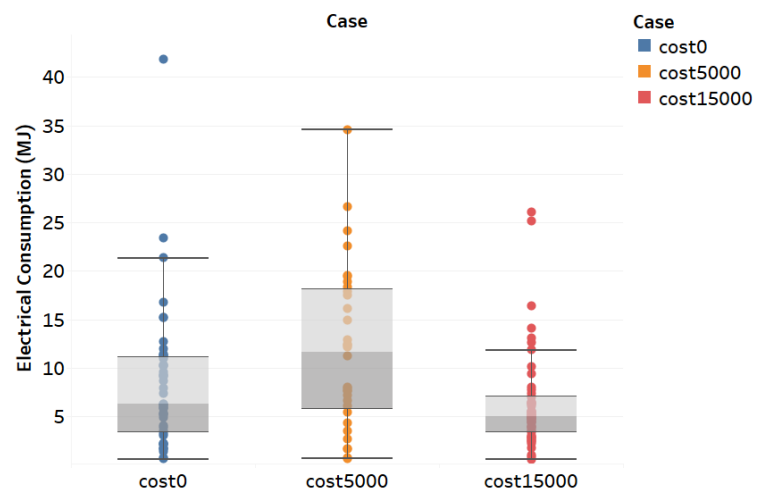

(b) Electrical Consumption box-plot for BEVs

Figure 8. Electrical Consumption distribution for BEVs.

A larger variation in electrical consumption for BEVs can be observed across the three different $\mathrm{CAV}$ penetration levels, showing substantial influence of the resultant penetration rates compared to the other powertrains. From Figure $8 \mathrm{a}$, it can be seen that there are three distinct trendlines that can be observed for unadjusted electrical consumption with respect to trip driving distance. In Figure $8 \mathrm{~b}$, the average electrical energy consumption across the different scenarios is further observed to vary and the variation is more apparent. This introduces a difference compared to what is observed for the other vehicle powertrains where no significant difference in fuel consumption is seen across the different $\mathrm{CAV}$ cost scenarios.

\section{Effect of Penetration Level on Fuel Consumed}

Figure 9 summarizes the distribution of driving distances and energy consumption for the different vehicle powertrains across different CAV scenarios.

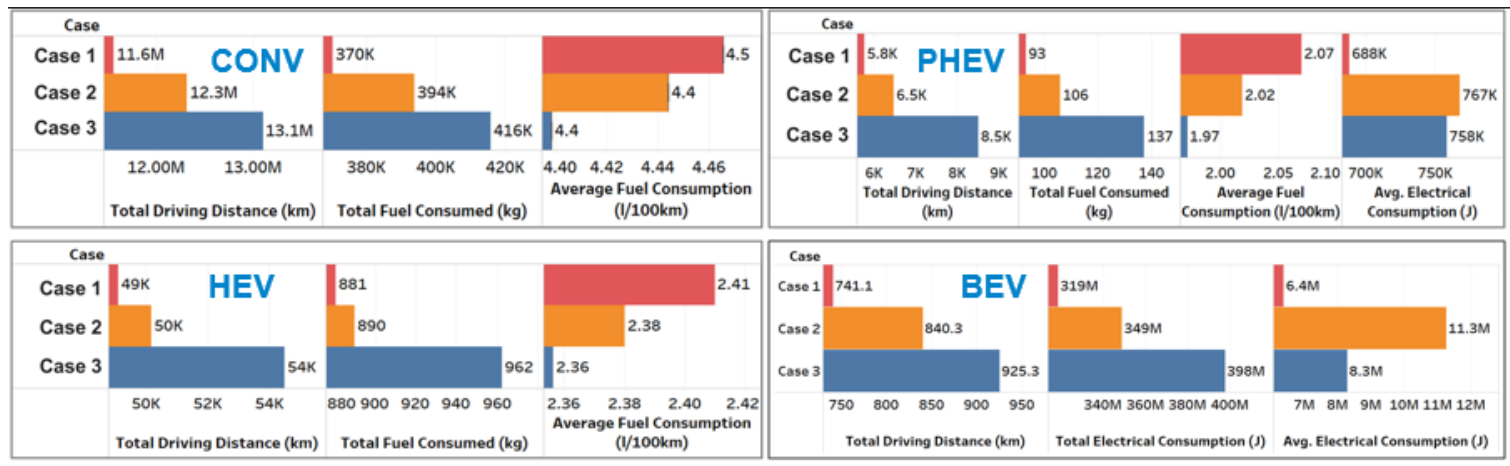

Figure 9. Simulation results summary across vehicle powertrains. 
The previous analysis showed some visual differences in the amount of fuel consumed for the various CAV penetration levels. It is unclear whether the primary contributor to this difference is in the resulting travel behavior through the miles traveled or the nature of each trip itself. An approach to isolating the effect of automation on total fuel consumed is to control for the distance traveled via regression methods. Also, it is of interest to understand whether certain powertrains benefit more (energy consumed) than others from the presence of more connected and automated vehicles on the road.

In this section, we focus on the total amount of energy consumed EnergyUsage for all simulated powertrains in Wh. Figure 10 shows a density plot of the log energy usage for the different powertrains. We note slight energy differences for conventional, hybrid, and plug-in hybrid vehicles, but energy consumption differences are more pronounced for battery electric vehicles.

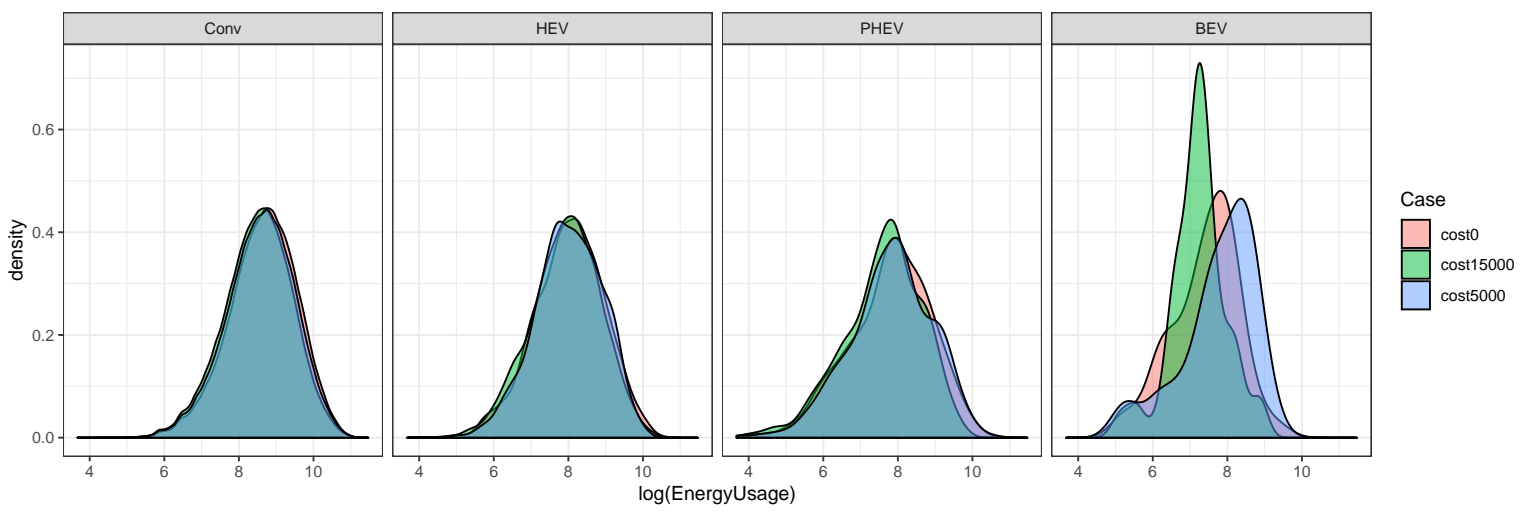

Figure 10. Density plot of log energy usage per powertrain.

The purpose of the log transform comes from the apparent gamma distributed response, which makes visual comparison difficult. We propose modeling the energy usage directly from the class of generalized linear models with a gamma density and an identity link. This approach naturally accounts for the skewness in the response and should avoid any potential heteroskedasticity of the residuals that would come from a Gaussian model. In Gaussian models for a response $Y$ we have $\mathbb{V}[Y]$ constant as a function of mean response $\mathbb{E}[Y \mid X]$ which in this setting is inappropriate [25]. As a matter of fact longer trips can vary in nature, with expected highway-like type driving. Further analysis also suggests, after conditioning on the trips distance, that the energy usage does have a close to gamma distribution.

We model each powertrain separately. The model has the form:

$$
g(\mathbb{E}[Y \mid X])=X^{T} \beta
$$

with $g$ an identity link function, $X$ the covariates of interest and $\beta$ the true parameters to estimate.

Figure 11 shows the resulting fit for conventional, HEV and BEV vehicles along with the penetration levels estimates. Estimates are typically computed using maximum likelihood methods. Full details can be found in [26]. 


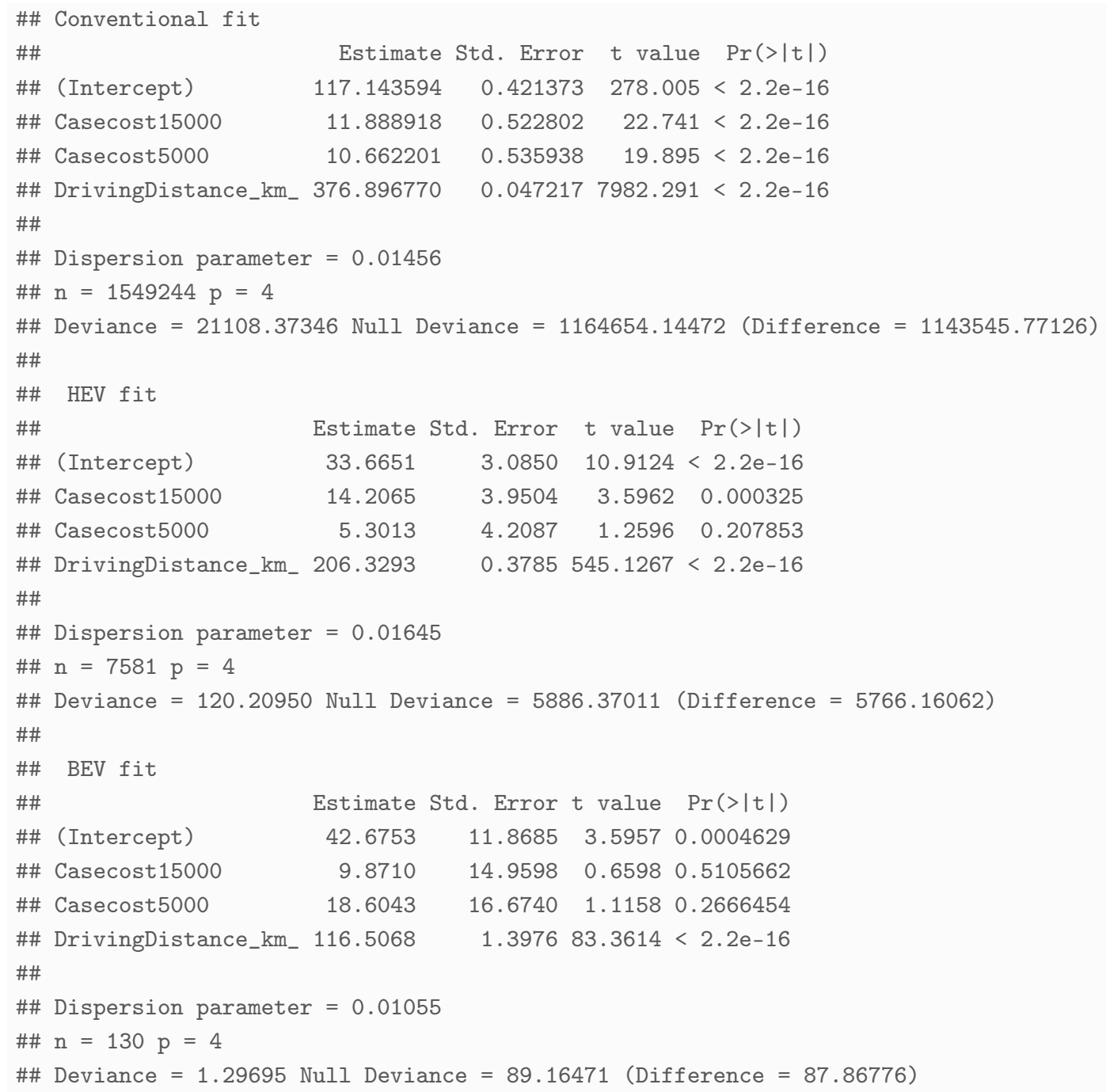

Figure 11. Resulting fit for different powertrains with respect to different penetration level estimates.

From the residual plots shown in Figure 12, we first note that a variance function going as the squared of the mean response could be too strong. This is especially true for conventional vehicles. Even so, the fit is appropriate and the estimates as well as the $t$ values can be trusted.
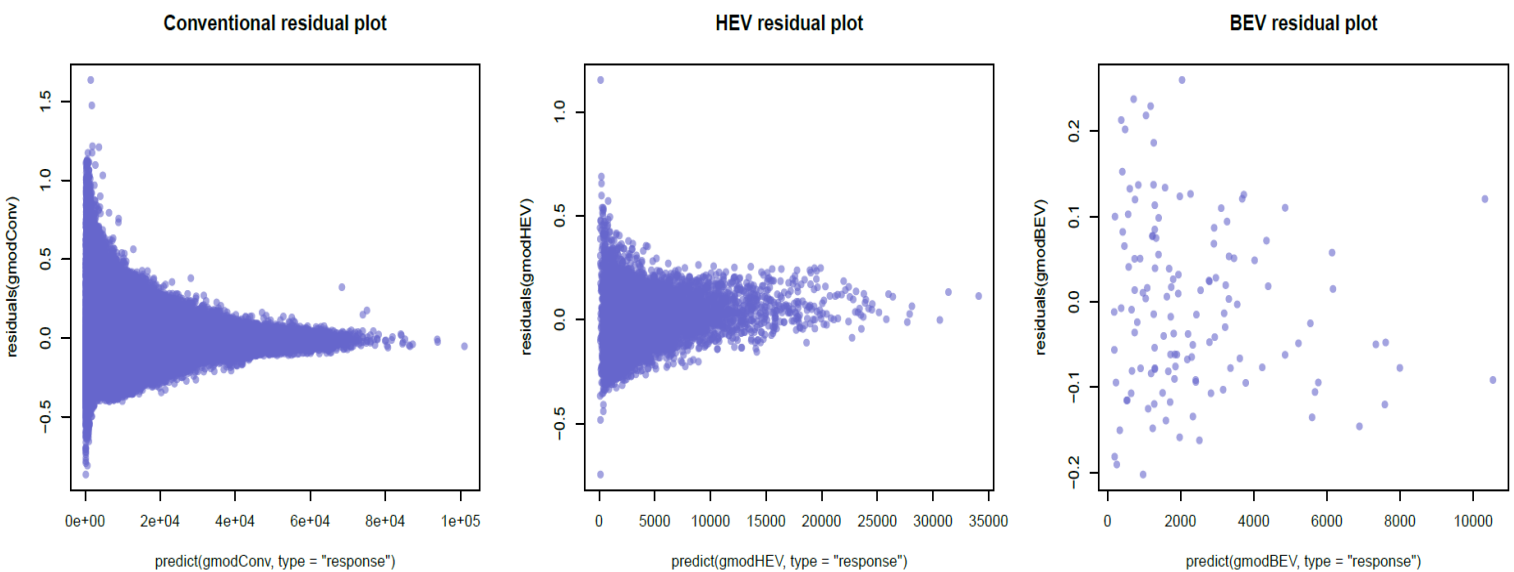

Figure 12. Residual plots. 
An interpretation of the results shows evidence for differences in energy levels for conventional vehicles in the various penetration scenarios. We note that an increase in automation cost leads to lower penetration levels. Consequently, this tends to increase overall energy consumed. Although the order of magnitude is not large (around 10 to 11 W.h additional on average), it is statistically significant enough to be reported as a notable difference. On the flip side, HEVs and BEVs are not as much affected by the penetration levels. In fact, in the case of an assumed automation cost of $\$ 5000$, the energy usage for HEVs is not affected enough to claim a change, suggesting that energy levels of HEVs are similar for $\$ 0$ or $\$ 5000$ of additional automation cost. However an additional automation cost of $\$ 15,000$ does impact HEV energy usage with a significant estimate of an additional $\sim 14$ W.h. Finally, BEVs seem not to differ for all levels, and this is reflected through the resulting high $p$-values of the estimates.

We emphasize that the power of this approach is that we managed to isolate energy differences on the penetration levels only by controlling for the trip distances. In fact, the outcome of this analysis is in contrast to the visual conclusion that one can draw by looking at Figure 10, especially for BEVs.

\section{Conclusions}

This study implemented a combined analysis of CAV energy impacts across different CAV cost scenarios. It demonstrates a powerful energy estimation tool for regional analysis that allows us to analyze the intersection between transport policy and vehicle technology. The purpose of the study is to evaluate the different impact of vehicle powertrains in energy consumption across the three cases studied.

With $\mathbf{1 0 0} \% \mathrm{CAV}$ penetration, the total driving distance for conventional powertrains increases by $13 \%$ and the average fuel consumption decreases by $1.5 \%$. For HEV powertrains, the total driving distance increases by $\mathbf{1 0} \%$ and the average fuel consumption decreases by $\mathbf{2 . 2} \%$. For PHEV powertrains, the total driving distance increases by $47 \%$ and the average fuel consumption decreases by $4 \%$, and for BEV powertrains, the total driving distance increases by $25 \%$ and the average electrical energy consumption increases by $33 \%$.

Through varied visual inspection, it can be seen that there is a minimum impact of the different vehicle powertrains across the three different $C A V$ cost cases that can be explained without taking driving distance into account. Further statistical analysis of the results, isolating the influence of the trip distances, shows some influence of the conventional vehicles in determining the overall energy consumption across the three different cost cases. With higher CAV penetration, and hence increasing average trip lengths, conventional vehicles tend to operate with better fuel efficiency and hence contributes to the overall energy consumption across the different cases.

In this study, there were no changes in the assumptions of the vehicle design itself across the three CAV penetration levels. Further research studies would be implemented to better model connected and automated vehicles, accounting for additional electrical accessory loads and vehicle dynamics for different levels of connectivity and automation in vehicles.

Author Contributions: Conceptualization, E.S.I. and A.R.; methodology, E.S.I. and A.R.; software, E.S.I.; validation, E.S.I. and A.R.; formal analysis, E.S.I., A.M. and N.K.; investigation, E.S.I. and A.R; resources, A.R.; data curation, E.S.I. and A.R.; writing-original draft preparation, E.S.I.; writing-review and editing, E.S.I.; visualization, E.S.I., A.M. and N.K.; supervision, A.R.; project administration, E.S.I. and A.R.; funding acquisition, A.R. All authors have read and agreed to the published version of the manuscript.

Funding: This research was funded by Vehicle Technologies Office I Office of Energy Efficiency and Renewable Energy | U.S. Department of Energy (DE-AC02-06CH11357)

Acknowledgments: This work was supported by the U.S. Department of Energy's Vehicle Technologies Office under the direction of Mr. David Anderson. The submitted manuscript has been created by UChicago Argonne, LLC, Operator of Argonne National Laboratory ("Argonne"). Argonne, a U.S. Department of Energy Office of Science laboratory, is operated under Contract No. DE-AC02-06CH11357. The views and opinions of the authors expressed herein do not necessarily state or reflect those of the United States Government or any agency thereof. Neither the United States Government nor any agency thereof, nor any of their employees, makes any warranty, expressed or implied, or assumes any legal liability or responsibility for the accuracy, completeness, or usefulness of any information, apparatus, product, or process disclosed, or represents that its use would not infringe privately owned rights. 
Conflicts of Interest: The authors declare no conflict of interest. The funders had no role in the design of the study; in the collection, analyses, or interpretation of data; in the writing of the manuscript, or in the decision to publish the results.

\section{Abbreviations}

The following abbreviations are used in this manuscript:

$\begin{array}{ll}\text { AER } & \text { All-Electric Range } \\ \text { ANL } & \text { Argonne National Laboratory } \\ \text { BEV } & \text { Battery-Electric Vehicle powertrain } \\ \text { CAV } & \text { Connected-Autonomous Vehicle } \\ \text { CONV } & \text { Conventional vehicle powertrain } \\ \text { HEV } & \text { Hybrid Electric Vehicle powertrain } \\ \text { PHEV } & \text { Plug-In Hybrid Electric Vehicle powertrain } \\ \text { U.S. DOE } & \text { United States Department of Energy } \\ \text { U.S. DOT } & \text { United States Department of Transportation }\end{array}$

\section{References}

1. Shidore, N.; Islam, E.; Vijayagopal, R.; Kim, N.; Moawad, A.; Rousseau, A. A Large Scale Simulation Process to Evaluate Powertrain Technology Targets with Real World Driving. In Proceedings of the the 29th Electric Vehicle Symposium (EVS29), Montreal, QC, Canada, 19-22 June 2016.

2. Polaris. Available online: https:/ / www.anl.gov/es/polaris-transportation-system-simulation-tool (accessed on 18 October 2019).

3. Auld, J.; Hope, M.; Ley, H.; Sokolov, V.; Xu, B.; Zhang, K. POLARIS: Agent-based modeling framework development and implementation for integrated travel demand and network and operations simulations. Transp. Res. Part C 2016, 64, 101-116. [CrossRef]

4. Autonomie. Available online: http:/ / www.autonomie.net (accessed on 18 October 2019).

5. Islam, E.; Moawad, A.; Auld, J.; Karbowski, D.; Rousseau, A. Impact of Advanced Vehicle Technologies on Energy Consumption for the City of Detroit Using Transportation System Simulations. In Proceedings of the the VPPC2017, Belfort, France, 11-14 December 2017.

6. Ramezani, H.; Shladover, S.E.; Lu, X.-Y.; Altan, O.D. Micro-Simulation of Truck Platooning with Cooperative Adaptive Cruise Control: Model Development and a Case Study. Transp. Res. Board 2018, 2672, 55-65. [CrossRef]

7. Laclair, T.; Gao, Z.; Wang, R.; Rios, T.J.; Sanyal, J.; Karthik, R.; Nugent, P.; Ravulaparthy, S.; Berres, A. Development of a Real-Time Mobility Control and Visualization System with Predictive Vehicle Speed Control for Connected and Automated Vehicles (CAVs). In Proceedings of the 32nd Electric Vehicle Symposium (EVS32), Lyon, France, 19-22 May 2019.

8. Colin, S.; Waraich, R.; Gopal, A.R.; Campbell, A.; Pozdnukhov, A. Modeling Plug-in Electric Vehicle Charging Demand with BEAM, the Framework for Behavior Energy Autonomy Mobility; Research Report; LBNL-2001018; Energy Technologies Area: Berkeley, CA, USA, 2017.

9. Auld, J.; Javanmardi, M.; Islam, E.; Karbowski, D.; Stephens, T.; Rousseau, A. Preliminary analysis of the impacts of CAV technologies on travel demand and energy use. In Proceedings of the the Automated Vehicles Symposium (AVS 2017), San Francisco, CA, USA, 11-13 July 2017.

10. Islam, E.; Moawad, A.; Kim, N.; Rousseau, A. An Extensive Study on Sizing, Energy Consumption and Cost of Advanced Vehicle Technologies; Report to the U.S. Department of Energy; Contract ANL/ESD-17/17; U.S. Department of Energy Office of Scientific and Technical Information: Oak Ridge, TN, USA, 2017.

11. Christopher, N.; Jessica, O.; Shladover, S.E.; Delphine, C. Cooperative Adaptive Cruise Control: Driver Acceptance of Following Gap Settings Less than One Second. Proc. Hum. Factors Ergon. Soc. Annu. Meet. 2010, 54, 2033-2037.

12. Tsugawa, S. An Overview on an Automated Truck Platoon within the Energy ITS Project. IFAC Proc. 2013, 46, 41-46. [CrossRef] 
13. Bonnet, C.; Fritz, H. Fuel Consumption Reduction in a Platoon: Experimental Results with Two Electronically Coupled Trucks at Close Spacing; SAE International: Warrendale, PA, USA, 2000; doi:10.4271/2000-01-3056. [CrossRef]

14. Browand, F.; McArthur, J.; Radovich, C. Fuel saving achieved in the field test of two tandem trucks. In California Partners for Advanced Transit and Highways; University of California: Berkele, CA, USA, 2004.

15. Alam, A.A.; Gattami, A.; Johansson, K.H. An experimental study on the fuel reduction potential of heavy duty vehicle platooning. In Proceedings of the 13th International IEEE Conference on Intelligent Transportation Systems, Madeira Island, Portugal, 19-22 September 2010; pp. 306-311.

16. Shida, M.; Nemoto, Y. Development of a small-distance vehicle platooning system. In Proceedings of the 16th ITS World Congress and Exhibition on Intelligent Transport Systems and Services, Stockholm, Sweden, 21-25 September 2009.

17. Eben Li, S.; Li, K.; Wang, J. Economy-oriented vehicle adaptive cruise control with coordinating multiple objectives function. Veh. Syst. Dyn. 2013, 51, 1-17. [CrossRef]

18. Shladover, S.; Su, D.; Lu, X.-Y. Impacts of Cooperative Adaptive Cruise Control on Freeway Traffic Flow. Transp. Res. Rec. J. Transp. Res. Board 2012, 2324, 63-70 [CrossRef]

19. Newell, G.F. A simplified theory of kinematic waves in highway traffic, part I: General theory. Transp. Res. Part B Methodol. 1993, 27, 281-287 [CrossRef]

20. Williams, C.A.; Mohammadian, A.; Auld, J.; Doherty, S.T. Enhancing Traveler Context through Transferable Activity Patterns. In Mobile Computing, Applications, and Services. MobiCASE 2012. Lecture Notes of the Institute for Computer Sciences, Social Informatics and Telecommunications Engineering; Uhler, D., Mehta, K., Wong, J.L., Eds.; Springer: Berlin/ Heidelberg, Germany, 2013; Volume 110.

21. Auld, J.; Mohammadian, A.; Roorda, M.J. Implementation of Scheduling Conflict Resolution Model in Activity-Scheduling System. Transp. Res. Rec. J. Transp. Res. Board 2009, 2135, 96-105. [CrossRef]

22. Bansal, P.; Kockelman, K.; Singh, A. Assessing public opinions of and interest in new vehicle technologies: An Austin perspective. Transp. Res. Part C 2016, 67, 1-4. [CrossRef]

23. Shabanpour, R.; Golshani, N.; Auld, J.; Mohammadian, A. Willingness-to-pay for automated vehicles technology: A random parameters and random thresholds HOPIT model. In Proceedings of the International Choice Modelling Conference 2017, Cape Town, South Africa, 3-5 April 2017.

24. Islam, E.S.; Moawad, A.; Kim, N.; Rousseau, A. A Detailed Vehicle Simulation Process to Support CAFE Standards; Argonne National Lab Report ANL/ESD-18/6; Supporting Document no. NHTSA-2018-0067-0007; NHTSA: Washington, DC, USA, 2018.

25. Faraway, J.J. Extending the Linear Model with $R$ (Texts in Statistical Science); Chapman \& Hall/CRC: Boca Raton, FL, USA, 2005

26. McCullagh, P.; Nelder, J.A. Generalized Linear Models, 2nd ed.; Chapman \& Hall: Boca London, UK, 1989. 